\title{
Analysis of arc pressure effect on metal transfer in gas-metal arc welding
}

\author{
Sehun Rhee and Elijah Kannatey-Asibu, Jr. \\ Department of Mechanical Engineering and Applied Mechanics, The University of Michigan, Ann Arbor, \\ Michigan 48109
}

(Received 9 November 1990; accepted for publication 1 August 1991)

\begin{abstract}
Metal transfer has been analyzed using both the static force balance method and the pinch instability theory. The influences of arc pressure as well as electromagnetic pressure in the development of instability in the cylindrical molten metal were considered. Using perturbation theory, the dispersion equation was obtained in terms of the arc current density, and the drop diameters were then calculated. The drop diameters, which were determined by considering the arc effect, were found to be greater than those drop diameters that were obtained without the arc effect. The transition current was obtained on the assumption that necking starts when the arc covers an entire drop, at which point spray transfer occurs as the molten droplet becomes cylindrical in shape. This explains why spray transfer or transition does not appear with carbon dioxide or helium shielding gas since the arc then exists only at the lower half of the droplet.
\end{abstract}

\section{INTRODUCTION}

The phenomenon of liquid column instability was apparently first investigated by Rayleigh ${ }^{1}$ when he postulated that instability began when the wavelength was greater than the circumferential length. Dattner, Lehnert, and Lundquist ${ }^{2}$ observed the instability experimentally in a column of current-carrying mercury, and Murty ${ }^{3}$ developed the theory of the pinch instability phenomenon in an axial electric current field. The pinch instability theory was first applied to welding metal transfer by Lancaster. ${ }^{4}$ Allum $^{5}$ then further investigated the pinch instability of the molten metal transfer and determined the drop diameters and their initial velocities. However, the influence of the arc pressure on the transfer mechanism has thus far not been investigated.

In gas-metal arc welding (GMAW), a molten drop is transferred from the electrode tip to the weld pool by the forces acting on the droplet. The transfer can be either globular or spray. Transition between globular transfer and spray transfer was first observed in gas-metal arc welding using argon as the shielding gas by Lesnewich. ${ }^{6}$ The metal transfer mechanism has been studied by the static force balance method, which is based on the concept that a drop detaches when the total detaching forces acting on the droplet exceed the surface tension force, which is the retaining force. ${ }^{7}$ Greene $^{8}$ determined the electromagnetic force assuming a spherical molten drop and further assuming that the active area was occupied by the anode spot, and Amson $^{9}$ extended this to any axisymmetric molten drop. Needham, Cooksey, and Milner ${ }^{10}$ postulated that the gas drag was significant in the dynamics of the molten drop. The electromagnetic pressure was analyzed by Alov ${ }^{11}$ considering current flow along a cylindrical conductor. Waszink and Graat ${ }^{12}$ have determined the acting forces in the range of 100-200 A in plasma GMAW.

Lesnewich ${ }^{6}$ measured the drop frequency and observed an abrupt increase in the critical current region, which he called transition. Various criteria have since been developed for identifying the transition region. $\mathrm{Ma}$ and $\mathrm{Apps}^{13}$ postulated that an arc did not exist in the neck above the droplet since that portion was plastically deformed. Earlier, Waszink and Van Den Heuvel ${ }^{14}$ had suggested that the transition to spray transfer occurred when the sum of the elcctromagnetic force and the force exerted by the incoming liquid exceeded the surface tension force. Their criterion was based on fluid flow in a pipe and served as a good basis for validating the experimental results of Lesnewich. ${ }^{6}$ However, metal transfer in GMAW involves melting and thus is a vastly different mechanism from liquid flow within a pipe. Their criterion, for example, cannot explain the fact that transition does not occur in carbon dioxide or helium even at high currents.

Previous research on the pinch instability theory as applied to welding does not consider the effect of arc pressure. In this paper, the work of Murty, ${ }^{3} \mathrm{I}$ ancaster, ${ }^{4}$ and Allum ${ }^{5}$ is extended to incorporate the effect of arc pressure on the mechanics of spray transfer using the pinch instability theory. The forces acting on the molten drop and the drop diameters are also determined using the static force balance method. Finally, a model is proposed and used in determining the transition between globular and spray transfer. First, the works of Murty and Lancaster are briefly reviewed. This is followed by an analysis that considers the arc pressure effect. The point at which transition from globular to spray transfer occurs is then analyzed. A brief outline of the experimental procedure used to verify the analyses is then presented, followed by a discussion of the results.

\section{ANALYSIS}

There are two complementary theories for studying the metal transfer mechanism. One is the pinch instability method and the other is the static force balance method. The latter is appropriate for transfer at relatively low currents, while the former is more suitable for analyzing transfer after transition, i.e., at high currents. This study pre- 
sents extensions to the pinch instability theory as applied to welding by considering the influence of arc pressure on metal transfer.

\section{A. Pinch instability theory \\ 1. Background}

The equations governing flow of an inviscid currentcarrying incompressible fluid are given by the momentum, continuity, and Maxwell equations,

$$
\rho \frac{d v}{d t}=-\nabla P+\mathbf{J} \times \mathbf{B}
$$

or

$$
\rho \frac{\partial v}{\partial t}+\rho v \cdot \nabla v=-\nabla P+\mathbf{J} \times \mathbf{B},
$$

and

$$
\boldsymbol{\nabla} \cdot \boldsymbol{v}=0,
$$

where $v$ is the velocity, $\rho$ the density of the molten electrode, $P$ the pressure in the molten cylindrical droplet at the electrode tip, $\mathbf{J}$ the current density, $\mathbf{B}$ the magnetic-flux density, and $t$ the time.

When current flows along the conductor, the properties of the resulting electric and magnetic fields are as follows:

$$
\begin{aligned}
& \mu_{0} \mathbf{J}=\nabla \times \mathbf{B}, \\
& \frac{\partial \mathbf{B}}{\partial t}+\nabla \times \mathbf{E}=0, \\
& \mathbf{J}=\sigma(\mathbf{E}+v \times \mathbf{B}), \\
& \nabla \cdot \mathbf{J}=0, \\
& \nabla \cdot \mathbf{B}=0, \\
& \nabla \times \mathbf{J}=0,
\end{aligned}
$$

where $\mu_{0}$ is the permeability in free space, $\mathbf{E}$ the electricfield intensity, and $\sigma$ the electrical conductivity. The current density is expressed as a predefined scalar function $\psi$,

$$
\mathbf{J}=J_{0} \mathbf{e}_{\mathbf{z}}+\epsilon \nabla \psi,
$$

where $e_{z}$ is a unit vector in the axial direction, $J_{0}$ is the average current density in the axial direction, and $\epsilon$ is a perturbation parameter. Taking the divergence of each side of Eq. (9) gives

$$
\nabla^{2} \psi=0 \text {. }
$$

The solution of this Laplace equation for a cylindrical coordinate system is the modified Bessel function

$$
\psi=c I_{0}(k r) \sin (k z) \text {, }
$$

where $c$ is a constant, $k$ a wave number, $I_{0}$ the modified Bessel function of the first kind of order zero, and $r$ and $z$ represent the radial and axial coordinates of a cylindrical coordinate system. Since the component of current normal to the perturbed boundary is negligible, we have

$$
(\mathbf{J} \cdot \mathbf{n})_{b}=0 \text {, }
$$

where the subscript $b$ denotes the boundary, and $\mathbf{n}$ is the normal to the boundary. The three components of current and magnetic-flux densities in the radial $r$, circumferential $\theta$, and axial $z$ directions are then given by

$$
\begin{aligned}
& J_{r}=-\epsilon k J_{0}\left[I_{1}(k r) / I_{1}(k R)\right] \sin (k z), \\
& J_{\theta}=0, \\
& J_{z}=J_{0}-\epsilon k J_{0}\left[I_{0}(k r) / I_{1}(k R)\right] \cos (k z), \\
& B_{r}=B_{z}=0, \\
& B_{\theta}=-\epsilon \mu_{0} J_{0}\left[I_{1}(k r) / I_{1}(k R)\right] \cos (k z)+\left(\mu_{0} J_{0} r / 2\right),
\end{aligned}
$$

where $I_{1}$ is the modified Bessel function of the first kind of order one, $R$ is equilibrium radius of the molten metal, and $\mu_{0}$ is permeability. The liquid boundary radius $R_{s}$, is perturbed about a fixed radius $R$ with the perturbation parameter $\epsilon$,

$$
R_{s}=R+\epsilon \cos (k z)=R+\epsilon_{0} \cos (\omega t) \cos (k z),
$$

where $\omega$ is angular frequency and $\epsilon_{0}$ is the amplitude of the perturbation parameter.

\section{Model extension}

To determine the pressure distribution, it is necessary to know the pressure at the perturbed boundary. 'The total pressure at the boundary $P_{b}$, is determined by the surface tension of the liquid and the arc pressure outside the boundary,

$$
P_{b}=P_{1}+P_{2}+P_{a}
$$

where $P_{1}$ and $P_{2}$ are the pressures due to surface tension attributed to the cylindrical radius and the radius of curvature of the liquid wave, respectively, and $P_{a}$ is the pressure of the outer arc, neglecting the effect of plasma flow. These are given by

$$
\begin{aligned}
& P_{1}=\frac{\gamma}{R_{1}}=\frac{\gamma}{R}\left(1-\frac{\epsilon_{0}}{R} \cos (\omega t) \cos (k z)\right), \\
& P_{2}=\gamma / R_{2}=\epsilon_{0} k^{2} \gamma \cos (\omega t) \cos (k z), \\
& P_{a}=\left(\mu_{0} J_{a}^{2} / 4\right)\left[R_{a}^{2}-R^{2}-2 \epsilon_{0} R \cos (\omega t) \cos (k z)\right],
\end{aligned}
$$

where $R_{1}$ and $R_{2}$ are the cylindrical radius and radius of curvature for the surface wave, respectively, $J_{a}$ and $R_{a}$ are the arc current density and radius, respectively, and $\gamma$ is surface tension. Substituting Eqs. (20), (21), and (22) into (19), the total pressure on the boundary is obtained as

$$
\begin{aligned}
P_{b}= & \frac{\gamma}{R}+\frac{\mu_{0} J_{a}^{2}}{4}\left(R_{a}^{2}-R^{2}\right)+\frac{\gamma \epsilon_{0}}{R^{2}} \\
& \times\left(k^{2} R^{2}-1-\frac{\mu_{0} J_{a}^{2} R^{3}}{2 \gamma}\right) \cos (\omega t) \cos (k z) .
\end{aligned}
$$

The schematic of liquid flow and the arc is shown in Fig. 1. Defining $\alpha$ as the ratio of arc to the liquid current density, and $\beta$ as the ratio of arc to liquid radius, i.e., 


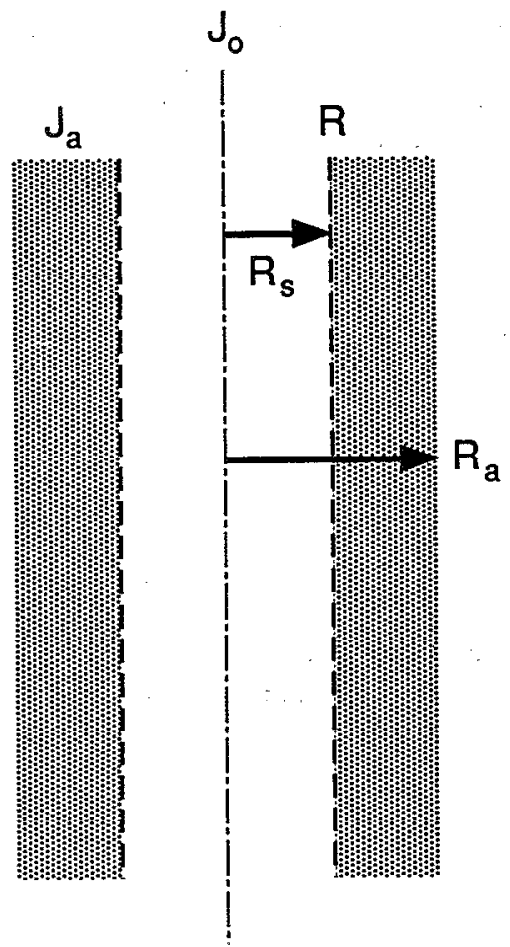

FIG. 1. Schematic of molten metal flow and the arc.

$$
J_{a}=\alpha J_{0} \text { and } R_{a}=\beta R \text {, }
$$

gives

$$
J_{0}=\frac{I}{\alpha \pi R^{2}\left(\beta^{2}-1\right)+\pi R^{2}}
$$

and

$$
J_{a}=\frac{\alpha I}{\alpha \pi R^{2}\left(\beta^{2}-1\right)+\pi R^{2}},
$$

where $I$ is the current.

Taking the divergence of the momentum equation (1), and combining with the continuity equation (2), results in the following equation:

$\nabla^{2} P=-\mu_{0} J_{0}^{2}+2 \epsilon k \mu_{0} J_{0}^{2}\left[I_{0}(k r) / I_{1}(k R)\right] \cos (k z)$.

Solving this equation with the perturbed boundary conditions (23) obtained above, the pressure distribution $P$ is obtained as

$$
\begin{aligned}
P= & \frac{\gamma}{R}+\frac{\mu_{0} J_{a}^{2}}{4}\left(R_{a}^{2}-R^{2}\right)+\frac{\mu_{0} J_{a}^{2}}{4}\left(R^{2}-r^{2}\right) \\
& +\epsilon_{0}\left\{\frac { I _ { 0 } ( k r ) } { I _ { 0 } ( k R ) } \left[\frac{\gamma}{R^{2}}\left(k^{2} R^{2}-1-\frac{\mu_{0} J_{a}^{2} R^{3}}{2 \gamma}\right)\right.\right. \\
& \left.\left.-\frac{\mu_{0} J_{0}^{2} R}{2}\right]+\mu_{0} J_{0}^{2} R \frac{I_{1}(k r)}{I_{1}(k R)}\right\} \cos (\omega t) \cos (k z) .
\end{aligned}
$$

Substituting Eq. (18) into the momentum equation (1) for the boundary, gives

$$
-\rho \omega^{2} \epsilon \cos (k z)=-\frac{d p}{d r}+(\mathbf{J} \times \mathbf{B})_{r}
$$

Using Eqs. (13)-(17), (27), and (28), the following eigenvalue equation is obtained:

$$
\begin{aligned}
\omega^{2}= & \frac{\gamma}{\rho R^{3}} x\left(x^{2}-1-\frac{\mu_{0} J_{a}^{2} R^{3}}{2 \gamma}\right) \frac{I_{1}(x)}{I_{0}(x)}-\frac{\mu_{0} J_{0}^{2}}{2 \rho} \\
& \times\left[2+x\left(\frac{I_{1}(x)}{I_{0}(x)}-\frac{I_{0}(x)}{I_{1}(x)}\right)\right],
\end{aligned}
$$

where $x$ is the dimensionless wave number and is defined by

$$
x=2 \pi R / \lambda=k R,
$$

where $\lambda$ is the wavelength of the perturbed boundary. To gain physical insight into the pinch instability of a liquid column, Eq. (18) can be expressed in a more general form as

$$
R_{s}=R+\epsilon_{0} \operatorname{Re}\left(e^{i \omega t}\right) \cos (k z),
$$

where $R_{s}$ is the liquid surface radius. When $\omega$ from Eq. (29) is real, i.e., $\zeta$, then

$$
R_{s}=R+\epsilon_{0} \cos (\xi t) \cos (k z),
$$

and in this case, since the perturbed function is periodic, the system is stable. When $\omega$ is imaginary, i.e., ij, then

$$
R_{s}=R+\epsilon_{0} e^{-\zeta t} \cos (k z) \text {. }
$$

In this case the perturbed function increases or decreases exponentially with time, and hence this system is unstable. At a critical value of $x$ for stability, $\omega^{2}$ is equal to zero, i.e.,

$$
\omega^{2}=0 \text { at } x=x_{c}
$$

where $x_{c}$ is the dimensionless wave number at which the angular frequency is zero.

To determine the value of $x$ corresponding to the maximum value of $\omega^{2}$, the derivative of $\omega^{2}$ with respect to $x$ is set to zero:

$$
\begin{aligned}
\frac{d \omega^{2}}{d x}= & \frac{\gamma}{\rho R^{3}} x\left[x^{2}-1-\frac{\mu_{0} J_{a}^{2} R^{3}}{2 \gamma}+2 x \frac{I_{1}}{I_{0}}\right. \\
& \left.-\left(x^{2}+1-\frac{\mu_{0} J_{a}^{2} R^{3}}{2 \gamma}\right) I \frac{1}{I_{0}^{2}}\right]-\frac{\mu_{0} J_{0}^{2}}{2 \rho}\left[-2 \frac{I_{0}}{I_{1}}\right. \\
& \left.+x\left(\frac{I_{0}^{2}}{I_{1}^{2}}-\frac{I_{1}^{2}}{I_{0}^{2}}\right)\right]=0 \text { at } x=x_{m}
\end{aligned}
$$

where $x_{m}$ is the dimensionless wave number at which the square of the angular frequency is maximum. This equation is a function of $x$, and $x_{m}$ is between 0 and $x_{c}$ (Fig. 2). Using a computational half-interval iteration method, the dimensionless wave numbers corresponding to the $\omega$ values at which the droplets form are calculated.

The total volume moved along the liquid cylinder per wavelength is assumed to be equal to the drop volume which is given by

$$
\lambda_{m} \pi R^{2}=(\pi / 6) d^{3}
$$




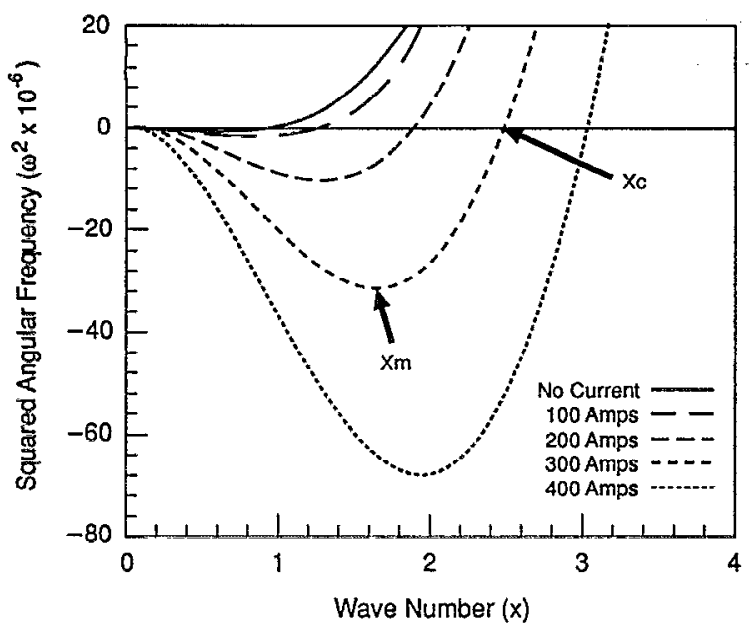

FIG. 2. Current effect on dimensionless wave number with parameters of Table I and $\alpha=0$.

where $\lambda_{m}$ is the wavelength corresponding to the dimensionless wave number for maximum values of $\omega^{2}$, and $d$ is the drop diameter. The drop diameter can thus be expressed in terms of the dimensionless wave number based on the maximum value of $\omega^{2}$,

$$
d=\left(12 \pi R^{3} / x_{m}\right)^{1 / 3} \text {. }
$$

\section{B. Transition current}

As the welding current is increased, a point is reached when a transition occurs from globular transfer to spray transfer. ${ }^{6}$ Waszink and Van Den Heuvel ${ }^{14}$ analyzed the transition current using the capillary tube effect and hence indicated that spray transfer occurred when the liquid flow velocity exceeded a critical velocity. However, this theory cannot explain the fact that transition does not occur when using carbon dioxide or helium shielding gases, even at very high currents. Allum's analysis ${ }^{5}$ is based on the pinch instability theory, and the resulting expression for the transition current essentially reduces to a proportionality with the square root of the surface tension force. In the following paragraph, we present a new criterion that results in better correlation with experimental results and also accounts for the effects of other shielding gases.

The angle $\theta$, which is the angle between the electrode axis and the point at which the arc contacts the droplet, is generally smaller than the angle $\phi$, which is the angle between the electrode axis and the point of contact of the droplet with the electrode in the lower current range. As the current increases, the angle $\theta$ also increases until it finally becomes equal to $\phi$. A new criterion is thus proposed in which necking, and thus transition, is considered to occur when the arc entirely covers the drop surface, i.e., $\theta=\phi$, and the transition current is determined on this basis. The rationale for developing this criterion is based on the fact that transition does not occur even at very high currents, when using carbon dioxide or helium as the shielding gas. In argon-carbon-dioxide mixtures, the arc locates under the molten droplet at low currents. However, the arc climbs as the current increases, and the solid begins to neck when the arc covers the entire droplet.

The transition current values were determined for specific wire radii and current densities in the molten droplet by considering the forces acting on the droplet and by using the static force balance equation discussed in Appen$\operatorname{dix}$ A. For an initial current $I_{\text {init }}, \theta$ and $\phi$ were determined iteratively by the half-interval method using equations (A7) and (A8). If $\theta$ was found to be less than $\phi$, the process continued with $I=I+1$ until $\theta=\phi$. That current value was then specified as the transition current.

\section{EXPERIMENTAL}

For verification of the preceding analyses, experiments were carried out using a gas-metal arc welding system with direct current reverse polarity in the flat position and with a constant current power source. The electrode was 1.6mm-diam mild steel and the workpiece a 6 -mm-thick mild steel plate. The welding speed was constant at $7.5 \mathrm{~mm} / \mathrm{s}$ with a wide range of currents and voltages from 150 to 400 $A$ and 26-32 V, respectively. Pure argon was the main shielding gas used, but carbon dioxide and helium gases were also used. The electrode extension was $14 \mathrm{~mm}$. A laser optical system (helium-neon laser; $5-\mathrm{mW}$ power, 632.6-nm wavelength) was used for shadowgraphing, and the metal transfer was observed using a high-speed motion analyzer with a film speed of 60 frames/s.

\section{RESULTS AND DISCUSSION}

\section{A. Pinch instability}

Equation (27) shows that the pressure distribution inside the perturbed liquid boundary depends on the distance in the radial direction, as well as the current density for the liquid and the arc. From the dispersion equation (29), the angular frequency for disturbances $\omega$ also depends on the current densities in the arc and liquid metal, as well as the material properties. In the absence of arc pressure, $J_{a}$ is equal to zero and this equation then reduces to that previously derived by Lancaster. ${ }^{4}$ When $\omega$ is real valued, the perturbation function is periodic and stable, and $\omega$ is then the angular frequency of the system. When $\omega$ is imaginary, the perturbed function varies exponentially. The relationship between the square of angular frequency for disturbances $\omega^{2}$ and dimensionless wave number $x$ is shown in Fig. 2, where the current effect is also shown. Figure 3 shows the arc current-density effect on the critical or maximum wave number. The data used for the plots are listed in Table I. As $x$ increases, the absolute value of $\omega^{2}$ increases to a maximum value, and then decreases, as shown in Figs. 2 and 3. When the absolute value of $\omega^{2}$ is maximum, the instability grows at a maximum rate and the fastest growing mode becomes the dominant one. ${ }^{15}$ The molten cylinder then breaks up into droplets.

The analysis also shows that the dimensionless wave number for the maximum angular frequency is greater for higher currents (Fig. 4). In other words, as the total axial current increases, $x_{m}$ also increases. That means either the wavelength at which the molten metal breaks up decreases 


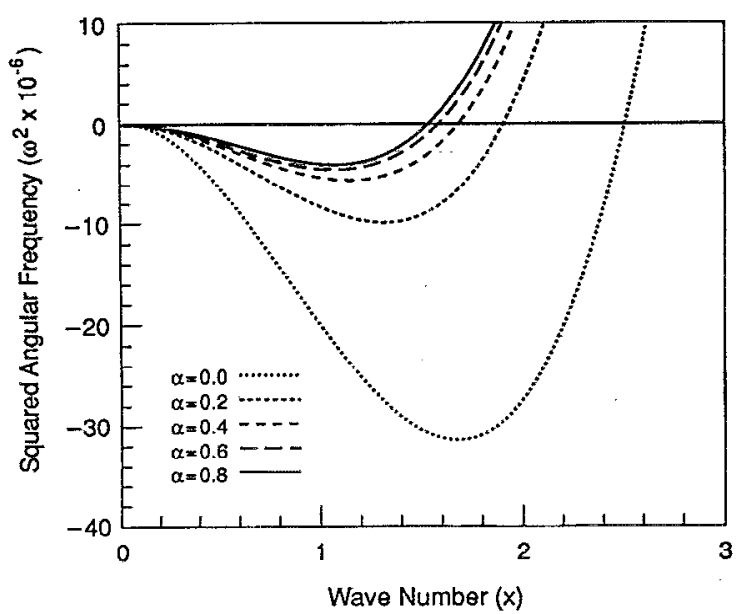

FIG. 3. Arc effect on dimensionless wave number with parameters of Table $I$ and $I=300 \mathrm{~A}$.

or the corresponding radius increases. However, since the droplet size is observed experimentally to decrease with increasing current, the former is more likely to be the case, i.e., the wavelength at breakup decreases with increasing current. Incorporating the arc pressure effect modifies the current-density and eigenvalue equations. For a given current, the dimensionless wave numbers for both the maximum and critical angular frequencies decrease as the current-density ratio $\alpha$ increases (Fig. 5). In other words, with an increasing proportion of the current flowing through the arc, the wavelength of molten metal breakup increases, resulting in an increasing droplet volume, which is again consistent with experimental observations. The drop diameters obtained from the wave numbers for maximum angular frequency are shown in Fig. 6. The experimental results indicate that a significant portion of the current flows through the arc, since the drop size for $\alpha=0.6$ correlates better with experiments than that for $\alpha=0$, i.e., when current flow through the arc is neglected. The drop diameters that would correspond to metal breakup at the critical $\left(x_{c}\right)$ rather than the maximum $\left(x_{m}\right)$ dimensionless wave number, and an $\alpha$ of 0.3 , are also shown for comparison, and it is evident that smaller drop diameters would result if breakup occurred just when the molten metal became unstable, and of course, the experimental results support the contention that breakup occurs when the instability grows at the maximum rate.

TABLE I. Input data for pinch instability.

\begin{tabular}{lc}
\hline \hline $\begin{array}{l}\text { Current } \\
\text { density (mild steel) }\end{array}$ & $250-400 \mathrm{~A}$ \\
\hline$\alpha$ & $7000 \mathrm{~kg} / \mathrm{m}^{3 \mathrm{a}}$ \\
\hline & $0-1$ \\
$J_{a}$ & 2 \\
$J_{0}$ & $0-350 \mathrm{~A} / \mathrm{mm}^{2}$ \\
$R_{a}$ & $440-1400 \mathrm{~A} / \mathrm{mm}^{2}$ \\
$R$ & $0.6 \mathrm{~mm}$ \\
\hline
\end{tabular}

"Reference 20.

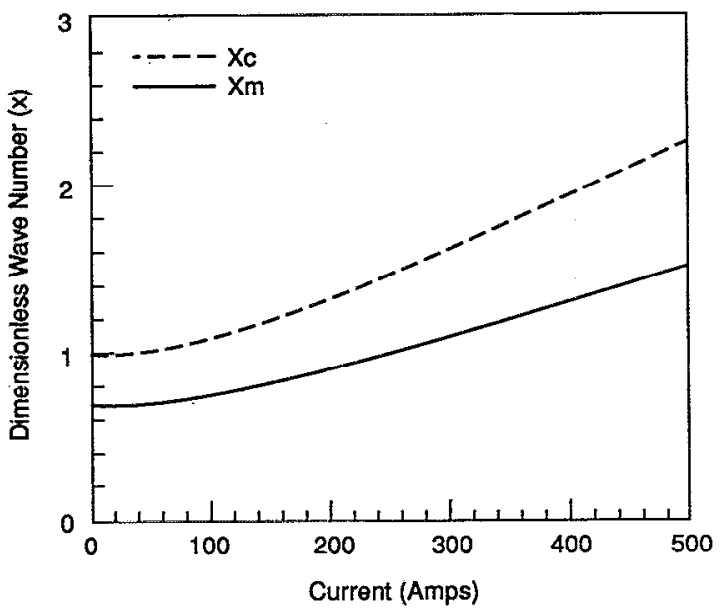

FIG. 4. Critical and maximum wave number with current values from Table I and $\alpha=0$.

The variation of drop diameters obtained by the static force balance method with current for different current densities is shown in Fig. 7 and indicates close agreement between experimental and analytical results for the lower current range in pure argon. It was found that the drop diameter was larger for high current density since the electromagnetic force decreased due to a reduction in the arc conduction area. From the experimental results, it is evident that the pinch instability theory is applicable in the higher current range while the static force balance method is more useful in the lower current range. Furthermore, incorporating the arc pressure effect results in better correlation of the pinch instability theory with experiment.

The theoretical results agree reasonably well with the results obtained experimentally for both the spray transfer region where the pinch instability theory was used, and the globular transfer region where the force balance method was used (Fig. 7). The results also show that increasing the current reduces the drop diameter. When compared with the results of Lancaster, which did not incorporate

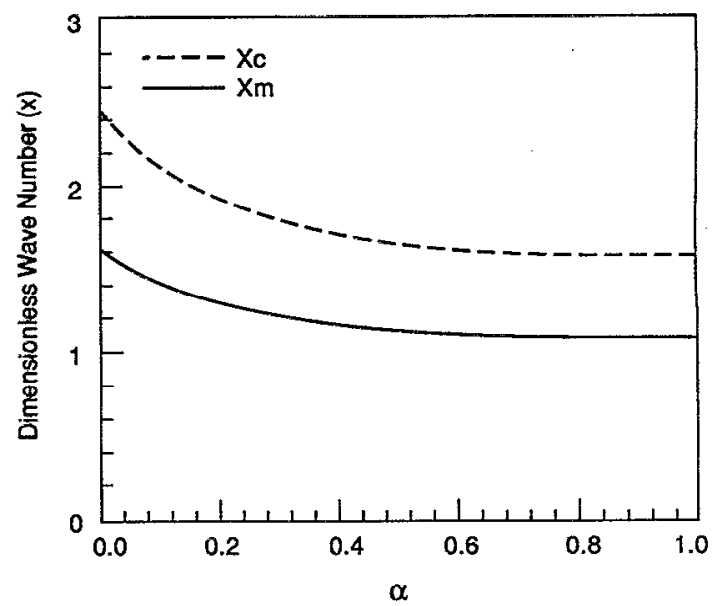

FIG. 5. Critical and maximum dimensionless wave number with $\alpha$ values from Table $I$ and $I=300 \mathrm{~A}$. 


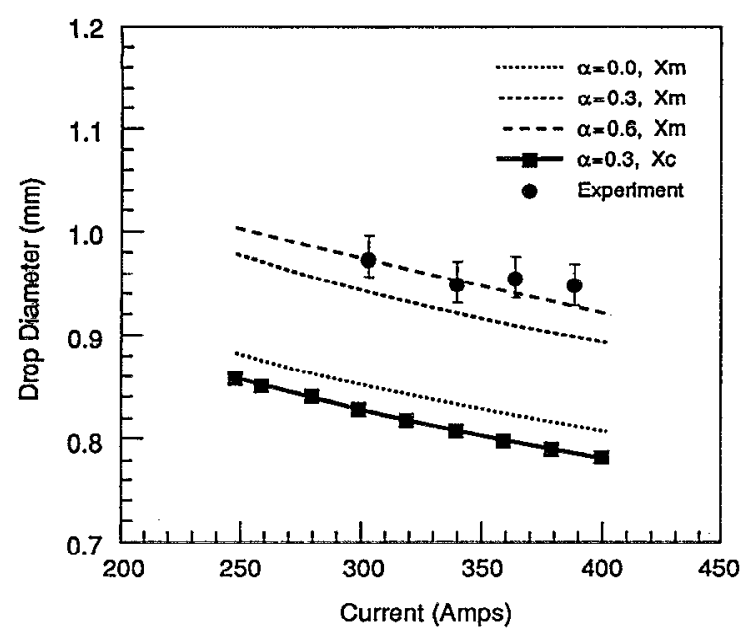

FIG. 6. Drop diameters from pinch instability theory with data values from Table I.

the arc pressure effect, the drop diameters obtained by the present analysis are larger as a result of current loss through the arc. It is also shown that considering the arc pressure effect results in better correlation with experiment.

In this analysis, we consider that all of the molten metal in one wavelength is detached with each drop, while Allum $^{5}$ assumed that some fluid remained attached to the wire, and therefore used $\frac{3}{4}$ of a wavelength as the amount of fluid detached in a drop. Using the latter assumption would result in drop diameters $10 \%$ smaller than our prediction and a poorer correlation with experiment.

The gravity term was neglected in Eq. (1) since the conditions under which instability occurs are such that the drop sizes and resulting drop weights are very small in comparison with the other terms in Eq. (1). At the lower current values at which the gravity term becomes significant, the drop shape is globular, and the instability model would not apply.

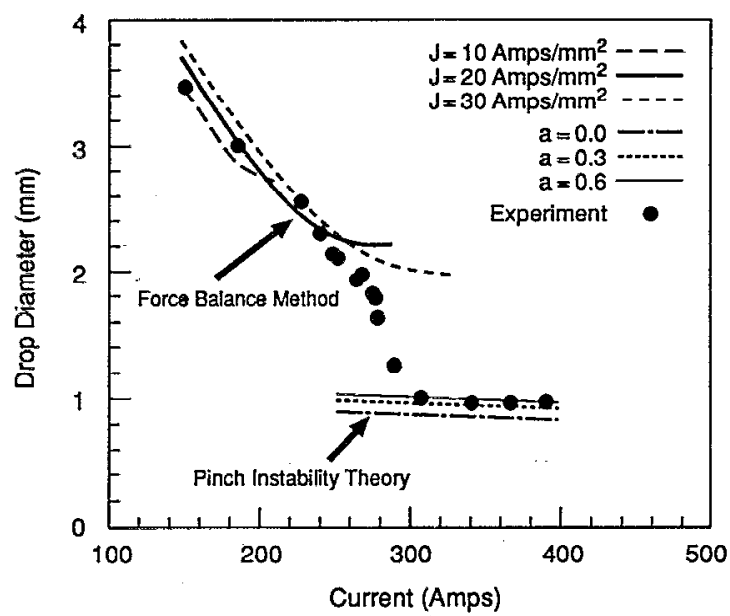

FIG. 7. Drop diameter variation with current, based on data from Tables I and II.
TABLE II. Input data for static force balance.

\begin{tabular}{lc}
\hline Gas temperature & $8000 \mathrm{~K}^{\mathrm{a}}$ \\
Gas density (argon) & $0.06 \mathrm{~kg} / \mathrm{m}^{3 \mathrm{~b}}$ \\
Gas velocity & $20.4+0.37 I+0.0004344 I^{2 \mathrm{c}}$ \\
Surface tension, $2500 \mathrm{~K}$ & $1.2 \mathrm{~N} / \mathrm{m}^{\mathrm{d}}$ \\
\hline \hline
\end{tabular}

${ }^{a}$ Reference 21.

${ }^{\mathrm{b}}$ Reference 20.

'Reference 10.

${ }^{\mathrm{d}}$ Reference 16.

\section{B. Transition}

At relatively low currents, the drop shape obtained experimentally was observed to be spherical, and under these conditions, metal transfer was globular. The forces that act on the droplet under these conditions are discussed in Appendix A, and the values used for determining the forces are shown in Table II. Due to difficulties in measuring the gas properties, approximate values were used. Lancaster ${ }^{16}$ postulated that the average gas velocity was half the maximum velocity measured by Needham and co-workers. ${ }^{10}$ The resulting forces required to detach the droplet are shown in Fig. 8. As the current increases, both the drag and gravitational forces decrease, while the electromagnetic force increases. However, the surface tension is almost constant since the temperature of the interface between the electrode tip and molten drop is the melting temperature.

With argon shielding, as the current increased, the size of the pendent drop decreased and the arc was observed to climb the droplet until it touched the solid tip or the interface between the electrode and the molten droplet. At that point, necking occurred. With pure carbon dioxide as the shielding gas, the arc does not cover the entire droplet due to the dissociation energy of carbon dioxide and likewise for helium, because of its high ionization energy. The high energy potential causes the current density to increase. The arc is thus always limited to the lower portion of the drop even at high currents, and that means there is

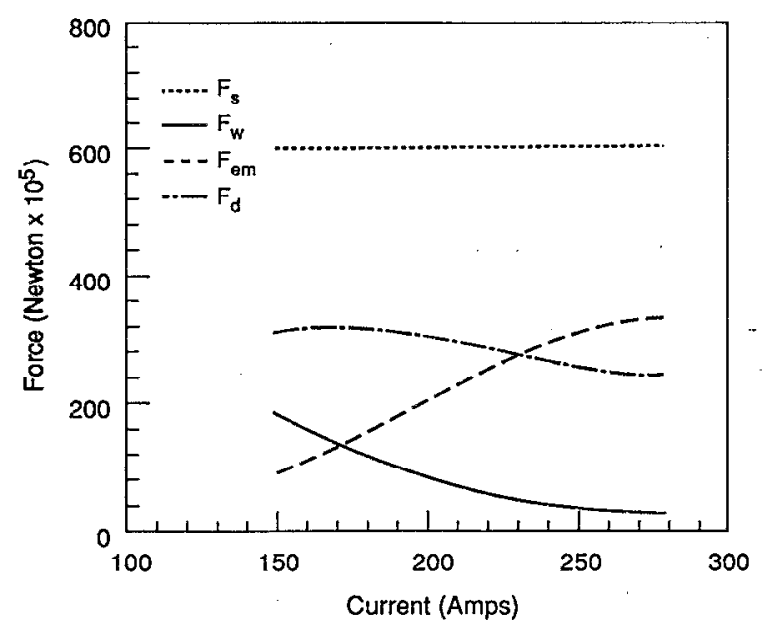

FIG. 8. Forces acting on the molten droplet, based on data from Table II and current density of $30 \times 10^{6} \mathrm{~A} / \mathrm{m}^{2}$. 


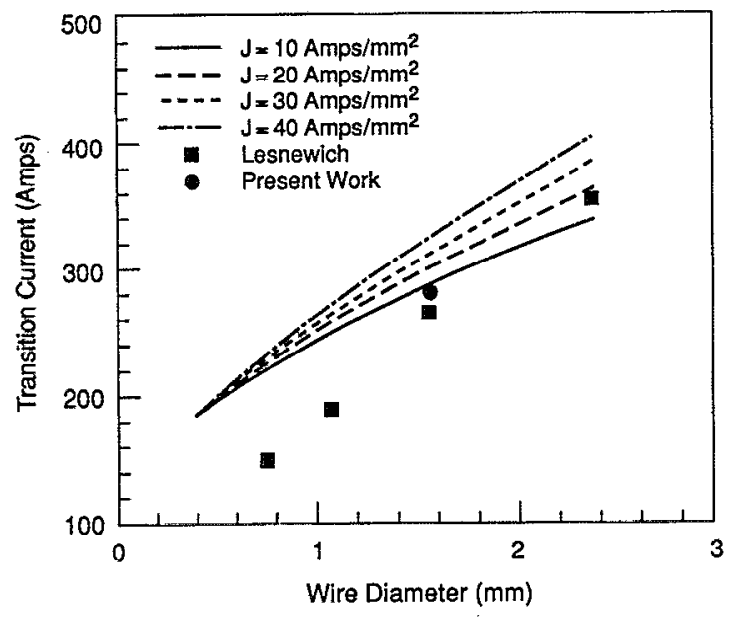

FIG. 9. Transition current variation with electrode diameter using values based on Table II.

no change in transfer mode. The transition current for various shielding gas mixtures is changed by the ionization energy; this is discussed in Appendix B.

The transition currents obtained using the proposed criterion, i.e., when the arc covers an entire droplet, are shown in Fig. 9 and compare well with the experimental results obtained by Lesnewich for mild steel in argon shielding gas. The transition current for the present experiment was found to be $280 \mathrm{~A}$ in argon shielding gas for a similar material of $1.6-\mathrm{mm}$-diam wire.

\section{CONCLUSIONS}

A modificd form of the dispersion equation based on the pinch instability theory is developed by considering the influence of arc pressure as well as the electromagnetic pressure within the liquid. The dimensionless wave number for the maximum absolute value of angular frequency is found to increase with increasing current and to decrease as the ratio of the arc current density to the liquid current density increases. Hence, the drop diameter obtained by considering arc pressure is greater than that without arc pressure and correlatcs better with experiments. In comparing the pinch instability theory with the static force balance method, the latter is found to be useful for globular transfer while the former is useful for spray transfer.

Transition from globular to spray transfer was identified as occurring when an arc covered the entire droplet and thus necking started. The transition currents so determined agreed reasonably well with the experimental results.

\section{APPENDIX A: FORCES ACTING ON THE DROPLET}

Greene $\mathrm{e}^{8}$ has derived the electromagnetic force $F_{\mathrm{em}}$ exerted on a spherical molten droplet

$$
\begin{aligned}
F_{\mathrm{em}}= & \frac{\mu_{0} \mathrm{I}^{2}}{4 \pi}\left(\ln \frac{\sin \theta}{\sin \phi}-0.25\right. \\
& \left.-\frac{1}{1-\cos \theta}+\frac{2}{(1-\cos \theta)^{2}} \ln \frac{2}{1+\cos \theta}\right),
\end{aligned}
$$

where $\mu_{0}$ is permeability, and $\theta$ and $\phi$ are the angle between the electrode axis and the point at which the arc contacts the droplet and the angle between the electrode axis and the point of contact of the droplet with the electrode, respectively.

The gravitational force $F_{w}$ and surface tension force $F_{s}$, are obtained from geometry as

$$
F_{w}=\left[\frac{4}{3} \pi R_{d}^{3}-\frac{1}{3} \pi h^{2}\left(3 R_{d}-h\right)\right] \rho g
$$

where

$$
h=R_{d}-\left(R_{d}^{2}-R_{w}^{2}\right)^{1 / 2}, \quad F_{s}=2 \pi R_{w} \gamma,
$$

where $R_{w}$ and $R_{d}$ are the wire and drop radii, respectively, $g$ is the gravitational acceleration, $\rho$ the density of the molten electrode, and $\gamma$ is the surface tension. The plasma drag force $F_{d}$ is obtained by the drag theory considering a gaseous fluid ${ }^{10}$ as

$$
F_{d}=0.5 C_{\mathrm{ds}} \rho_{g} v_{g}^{2} \pi R_{d}^{2}\left[1-\left(R_{w}^{2} / 2 R_{d}^{2}\right)\right]
$$

where $C_{\mathrm{ds}}$ is the drag coefficient for a sphere, and $\rho_{g}, v_{g}$, and $J$ are gas density, gas velocity, and current-density amplitude, respectively. The drag coefficient depends on the Reynolds number and is given ${ }^{17}$ by

$$
\begin{aligned}
& C_{\mathrm{ds}}=\frac{24}{R_{e}}+\frac{6}{\sqrt{1+R_{e}}}+0.4, \text { for } 0<R_{e}<200000, \\
& R_{e}=\rho_{g} v_{g} d / \mu,
\end{aligned}
$$

where $d$ is drop diameter, and $\mu$ is viscosity.

The droplet is in an equilibrium state when the resultant of the detachment forces is equal to that of the retaining forces, i.e.,

$$
F_{w}+F_{d}+F_{\text {em }}-F_{s}=0,
$$

where $F_{w}$ is the gravitational force, $F_{d}$ is the drag force exerted on the drop by the plasma, $F_{\text {em }}$ is the electromagnetic force due to current flow, and $F_{s}$ is a retaining force due to surface tension while the droplet is attached to the solid electrode. From the geometry of the arc conduction area,

$$
\cos \theta=1-\left(I / 2 \pi R_{d}^{2} J\right), \text { and } \sin \phi=R_{w} / R_{d} .
$$

The current conduction angle, i.e., the angle $\theta$ between the electrode axis and the radius to the point at which it is determined from Eqs. (A7) and (A8) using the drop diameter obtained by the static force balance method iteratively, again using the half-interval method since Eq. (A8) is a function of the drop diameter. $\phi$ was found to decrease slowly while $\theta$ increased rapidly as the current in- 
creased. Using $\theta$ and $\phi$, the magnitude of each force was also determined. Figure 8 shows the influence of current on each term.

\section{APPENDIX B: TRANSITION FOR ARGON-CARBON DIOXIDE MIXTURES}

The influence of shielding gas mixture on the transition current is first elucidated by determining the current density:

$$
J=\sigma E,
$$

where $\sigma$ is the electrical conductivity and $E$ is the electricfield intensity of the arc. The electrical conductivity can be obtained ${ }^{16}$ as

$$
\begin{aligned}
& \sigma=2.632 \times 10^{-2}\left(T^{1.5} / \ln Y\right), \\
& Y=1.25 \times 10^{7}\left(T^{1.5} / \sqrt{n_{e}}\right), \quad n_{e}=\delta n,
\end{aligned}
$$

where $T$ is the average absolute arc temperature, $n_{e}$ is the ionized electron number density, $n$ the number of molecules per unit volume, and $\delta$ is the degree of ionization. From Saha's equation, ${ }^{16}$

$$
\left[\delta^{2} /\left(1-\delta^{2}\right)\right]\left(P / P_{0}\right)=C T^{2.5} e^{-V / b T},
$$

where $b$ is Boltzmann's constant, $V$ is the ionization potential, $C$ is a constant, and $P$ and $P_{0}$ are pressure and standard atmospheric pressure, respectively. From known values of $T$ and $V, \delta$ is determined by interpolation from the graphical results of Lancaster: ${ }^{16}$

$$
\delta=5.6265 \times 10^{21} V^{-73.2454} T^{5.658 \nu^{0.37768}} .
$$

The ionization potentials of argon and helium are 15.7 and $24.6 \mathrm{eV}$, respectively. However, carbon dioxide has dissociation energy as well as ionization energy and thus the total energy is $31 \mathrm{eV}$. The total energy of an argoncarbon-dioxide gas mixture is obtained by interpolation, i.e.,

$$
V=15.3 h+15.7 \text {, }
$$

where $h$ is the ratio of carbon dioxide to argon. It is very difficult to measure the arc temperature accurately, but it is generally accepted that the arc temperature and electricfield intensity of an arc depend linearly on the ionization potential. ${ }^{19}$ Assuming an argon arc temperature of $8000 \mathrm{~K}$ and an electric-field intensity of $2500 \mathrm{~V} / \mathrm{m}$, the current density was obtained in terms of the ionization potential.

With the current density known, the transition current could then be determined for a 1.6-mm-wire diam; the results are shown in Fig. 10. The transition current was taken to be the average of the current value at which the drop frequency abruptly increases and the current value at which the frequency again levels off and was generally

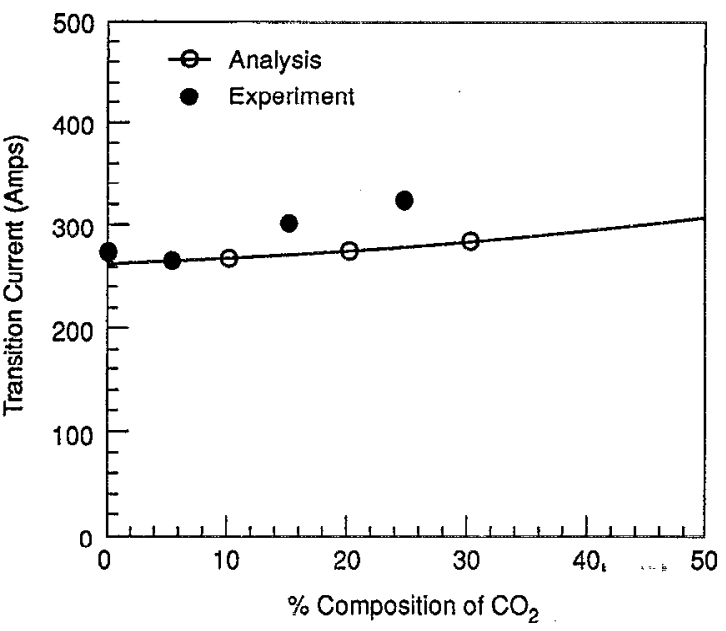

FIG. 10. Transition current for an argon-carbon-dioxide shielding gas mixture with other values from Table II.

found to increase with increasing carbon dioxide content. However, the $5 \% \mathrm{CO}_{2}$ mixture was found to have the lowest transition current, and this is likely due to the reduction in surface tension with surface oxidation, which tends to decrease the transition current. Its effect, however, becomes less significant with higher amounts of carbon dioxide with the result that the transition current then increases with increasing $\mathrm{CO}_{2}$ content.

${ }^{1}$ L. Rayleigh, Theory of Sound (Dover, New York, 1984), pp. 428-433.

${ }^{2}$ A. Dattner, B. Lehnert, and S. Lundquist, in Proceedings of the 2nd. UN Conference on the Peaceful uses of Atomic Energy, Geneva, 1958, Vol. 31, pp. 325-328.

${ }^{3}$ G. S. Murty, Arkiv foer Fysik 18, 241-250 (1960).

${ }^{4} \mathrm{~J}$. F. Lancaster, in Proceedings of the International Conference on Arc Physics and Weld Pool Behavior, 1979, pp. 170-174.

${ }^{5}$ C. J. Allum, J. Phys. D 18, 1431 (1985).

${ }^{6}$ A. Lesnewich, Weld. J. 37, 343s (1958).

${ }^{7}$ J. C. Amson, Br. Weld. J. 9, 232 (1962).

${ }^{8}$ W. J. Greene, AIEE 79, 194 (1960).

${ }^{9}$ J. C. Amson, Br. J. Appl. Phys. 16. 1169 (1965).

${ }^{10}$ J. C. Needham, C. J. Cooksey, and D. R. Milner, Br. Weld. J. 7, 101 (1960).

${ }^{11}$ A. A. Alov, The Welding Industry, 213 (1940).

${ }^{12}$ J. H. Waszink and L. H. J. Graat, Weld. J. 62, 108s (1983).

${ }^{13}$ J. Ma and R. L. Apps, Weld. Met. Fab. 50, 307 (1982).

${ }^{14}$ J. H. Waszink and G. J. P. M. Van Den Heuvel, Weld. J. 61, 269s (1982).

${ }^{15}$ P. G. Drazin and W. H. Reid, Hydrodynamic Stability (McGraw-Hill, New York, 1982), pp. 22-27.

${ }^{16}$ J. F. Lancaster, The Physics of Welding, 2nd ed. (Pergamon, New York, 1986), pp. 9-76.

${ }^{17}$ F. M. White, Viscous Flow (McGraw-Hill, New York, 1974), pp. 110 240.

${ }^{18}$ T. G. Cowling, Magnetohydrodynamics (Interscience, London, 1957), pp. 1-19.

${ }^{19}$ B. L. Ibatullin and V. F. Mukhin, Weld. Prod. 27, 53 (1980).

${ }^{20} \mathrm{X}$. Mondain-Monval (unpublished).

${ }^{21} \mathrm{~K}$. Acinger (unpublished). 